\title{
MULTIPLE INDEXING RELATIVISM ABOUT PREDICATES OF TASTE
}

\author{
DAN ZEMAN \\ University of Vienna \\ danczeman@gmail.com
}

SUMMARY: Focusing on predicates of taste, this paper puts forward a novel version of relativism, motivated by a recently discussed phenomenon: perspectival plurality. After showing that the phenomenon is problematic for at least some versions of relativism and discussing several possible answers on behalf of the relativist, I put forward my own version. The main feature of the proposal is the introduction in the index not of a single parameter for perspectives, but of a (possibly infinite) sequence of such parameters. In the last part of the paper, I defend the view against three objections.

KEY WORDS: semantics, perspectives, perspectival plurality, Lasersohn, "the paraphrasing strategy"

RESUMEN: Centrando la atención en los predicados de gusto, este artículo propone una nueva versión de relativismo, cuya motivación es un fenómeno que se ha discutido recientemente: la pluralidad de perspectivas. Tras mostrar que este fenómeno es problemático para algunas versiones de relativismo y discutir posibles respuestas que el relativista podría ofrecer, presento mi propia versión. La principal característica de la propuesta es la introducción en el índice, no de un único parámetro para perspectivas, sino de una secuencia (posiblemente infinita) de tales parámetros. En la última sección del artículo defiendo esta propuesta de tres objeciones.

PALABRAS CLAVE: semántica, perspectivas, pluralidad de perspectivas, Lasersohn, "la estrategia de paráfrasis"

Relativism about a variety of expressions has been very present in semantics lately — so much so that the view has become one of the main contenders in the debate over the right semantic account of those expressions. One type of expression that this debate has focused on is predicates of taste - expressions such as "fun", "tasty", "delicious", etc. Most authors dealing with such expressions agree that they are perspectival -in the sense that appeal to perspectives (or points of view, or standards, etc.) is needed for their semantic interpretation. Also, most authors agree that they give rise to a certain sort of context-sensitivity best exemplified by the fact that the same sentence containing such a predicate can have different truth values when uttered in different contexts. These two characteristics have been linked: the context-sensitivity of the sort described arises due to the perspectival character of predicates of taste. 
Canonically, relativism about predicates of taste has been cashed out as the view (or, more accurately, a cluster of views) that a parameter for perspectives should be introduced in the circumstances of evaluation/index ${ }^{1}$ with respect to which utterances of sentences containing predicates of taste are evaluated. The version of relativism I want to put forward in this paper goes further: according to what I call Multiple Indexing Relativism about predicates of taste, not only $a$ parameter for perspectives should be introduced in the index, but a sequence of such parameters. I will offer a sketch of the view. One motivation for proposing such a view is intrinsic: given that multiple indexing has been proposed in the past in connection with, for example, time and possible worlds, it would be interesting to see how such a view might look like as applied to predicates of taste. Another (perhaps better) motivation is that it provides a neat solution to a problem for (at least some versions of) relativism that has only recently been fully engaged with in the literature: that raised by the phenomenon known as "perspectival plurality". I will thus present this phenomenon and the problem it raises, and show how Multiple Indexing Relativism attempts to solve it. Finally, I address three possible objections to the view.

Before going further, two remarks. First, the main aim of this paper is to sketch and motivate a certain version of relativism about predicates of taste that is different from the canonical one; it is not to provide or even discuss arguments in favor of relativism per se. Given that discussion of such arguments has taken central stage in current literature, they are well-known ${ }^{2}$ and thus no rehearsing is needed. My contention in this paper is that if there are good arguments for relativism about predicates of taste, then the phenomenon of perspectival plurality gives you a reason to go further and be a Multiple Indexing Relativist. Second, although this paper deals

1 "Circumstances of evaluation" is the term used by Kaplan (1989); "index" is the term used by Lewis (1980). In what follows I will use "index" for convenience and for the sake of convergence with the phrase "multiple indexing", but the two terms should be taken (at least for current purposes) to be interchangeable.

${ }^{2}$ The argument from faultless disagreement has been put forward by, among others, Kölbel (2004a) and Lasersohn $(2005,2016)$. Retraction has been mainly the focus of MacFarlane's work, a comprehensive picture of which is given in his (2014) book. Eavesdropping scenarios have also been used to argue in favor of relativism — see, for example, Egan, Hawthorne and Weatherson (2005). Lasersohn (2009) uses arguments from de se readings of predicates of taste, their embedding under factive propositional attitude verbs like "recognize" and from truth evaluative adverbs like "correctly", while Kölbel (2009) tries out a version of the "operator argument" known from Kaplan (1989). 
with predicates of taste, I take the view proposed to apply to other perspectival expressions as well. But while I will refer to such expressions in relation to various issues in several footnotes, applying the view to those expressions might not be entirely straightforward.

The paper proceeds as follows. In section 1, I say a few words about predicates of taste and show how canonical relativism treats them. In section 2, I present the phenomenon of perspectival plurality and the problem it raises for (certain versions of) relativism. After presenting and criticizing some possible relativist solutions to the problem in a short section 3 , in the following section I offer a sketch of Multiple Indexing Relativism. In section 5, I deal with the three objections.

\section{Predicates of Taste and Relativism}

Predicates of taste are expressions like "tasty", "delicious", "disgusting", "fun", "boring", "cool", etc. Together with many others, ${ }^{3}$ they belong to a class that could be called "perspectival expressions", whose main purpose is to describe how people experience bits of reality from a certain perspective (a certain point of view, given a certain standard, etc.) or another. The use of perspectival expressions is important in a person's life because it allows both to communicate how things are from one's own perspective, but also to understand how things are from someone else's.

Predicates of taste are perspectival in the sense described. To see this, consider the following. If someone is asked whether licorice is tasty, the answer will be immediate. Most probably, the answer will be that licorice is tasty or not from the perspective of the speaker, even if perspectives are not explicitly mentioned in the exchange. Immediacy and implicitness, however, should not obscure the fact that, in answering the question, licorice is deemed tasty or not with respect to a perspective: the speaker's. But the speaker's perspective is not the only one that can matter: licorice can be deemed tasty or not from another person's perspective - as when, for example, the speaker talks from her child's point of view (such a use of "tasty" has been called "exocentric", in contrast to the first, "autocentric" use-

${ }^{3}$ Aesthetic predicates such as "beautiful", "ugly", "sublime"; moral predicates such as "good", "bad", or the moral "ought to"; epistemic modals such as "might" or "must"; epistemic terms such as "knows", "is justified", etc. have all been classified in this category. Surely, the sense of "perspective" relevant for each type of expression is different, but at this point I'm using the term in a neutral, intuitive sense (to be contrasted with the technical sense of the term to be introduced shortly). 
see Lasersohn (2005) and Stephenson (2007) for discussion). Alternatively, in a context in which it isn't clear which is the perspective that matters, asking whether licorice is tasty should be answered with another question: tasty to whom? All this seems to point to the same fact: that, in assessing whether something is tasty or not, a perspective needs to be supplied. "Tasty" is perspectival.

Another feature of predicates of taste is that they give rise to a certain form of context-sensitivity, illustrated by the fact that sentences in which they appear can vary in truth value when uttered in different contexts. Thus, suppose that Anne and Bob (two 8-year olds) taste licorice the first time. Anne loves it, but Bob is repelled by it. Assuming that each utter

\section{(1) Licorice is tasty}

in separate contexts, Anne's utterance of (1) is intuitively true in her context and Bob's utterance of (1) is false in his context. The truth value of (1) can thus vary with the context in which it is uttered.

The two characteristics brought to the fore above are linked. It is a natural thought that the context-sensitivity of sentences like (1) can be traced to the perspectivality of "tasty": Anne and Bob's utterances of (1) have different truth values when uttered in the two contexts because the perspective relevant for interpreting each utterance is different. From the point of view of a semantic theory -my focus here - this boils down to the task of capturing the context-sensitivity of sentences like (1) via the perspectivality of the predicates themselves. In other words, in order to capture the context-sensitivity of sentences like (1), a semantic theory has to find a place for perspectives in its apparatus. Various semantic theories situate perspectives in different places within their conceptual structure. Relativism about predicates of taste captures the context-sensitivity of sentences like (1) by introducing a parameter for perspectives in the index with respect to which utterances of such sentences are evaluated. This contrasts with other semantic views - for example contextualism, which captures the context-sensitivity of sentences like (1) by introducing perspectives directly in the content of utterances of such sentences. By introducing a parameter for perspectives in the index, relativism not only takes a stance on what such parameters should there be (possible worlds and perhaps other parameters plus perspectives), but also on the type of semantic content that utterances of sentences like (1) express in context (perspective-neutral contents). This is canonically expressed in a more formal manner as follows, with (2) giving the relativist truth-conditions of (1): 
(2) $[[\text { Licorice is tasty }]]^{c, w, p}=1$ iff licorice is tasty in $w$ according to $p$,

where " $c$ " stands for context, " $w$ " for possible worlds, " $p$ " for perspectives (other possible parameters are ignored for simplicity).

Now, relativism has been proposed in more than one form, and not everyone will take the view described above to amount to a relativist view worth its salt. One locus of variation pertains to the issue of which context it is that provides the values for the parameter for perspectives postulated. According to tamer versions, it is the context of utterance that provides the required values (as in the Kaplanian framework embraced by relativists like Kölbel (2004b) or Recanati (2007), among others); according to more radical versions, it is the "context of assessment" (MacFarlane 2014; Lasersohn 2005; 2016). Which version amounts to a "true" relativist view and which doesn't is, to a certain extent, a terminological issue. However, it is worth noting that the authors claiming that only the more radical version is the "true" version of relativism also postulate the relevant parameters in the index. The description above is thus accurate and can be used as the common denominator. It is also worth noting that, as it stands, the description is neutral on which is the context that provides the required values for the parameter for perspectives. A second locus of variation pertains to the issue of whose perspective is it that is relevant in a certain context. As we have already seen, that varies across contexts too. I thus adopt a flexible relativist view that allows the values for the parameter for perspectives to be those of any relevant person or group.

\section{Perspectival Plurality and the Problem It Raises for Relativism}

In the previous section, I showed that one of the main features of predicates of taste is their perspectivality, which plays a crucial role in any semantic theory's attempt to capture the context-sensitivity of sentences like (1). Recently, a related yet surprising phenomenon has been discussed in literature: what Kneer (2015) and Kneer, Vicente and Zeman (2017) have called "perspectival plurality". Perspectival plurality is the phenomenon whereby sentences containing two or more predicates of taste have interpretations (what I will call "plural readings") that require appeal to two or more perspectives. The phenomenon is interesting in its own right, but also relevant in connection to relativism because it raises a problem for at least some versions of it. To anticipate: the problem for relativism stems from its 
adoption (at least by some of its proponents) of a "one sentence-one perspective" principle that allows evaluation of utterances relative to at most one perspective.

As it happens, plural readings are not easily available without a certain amount of contextual setting. Consequently, I will provide such a setting before presenting the examples — with a more elaborated scenario for the first example, and with less elaborated ones for the others. Thus, imagine that Halloween has just passed, and several families from the neighborhood have gathered to talk about how they spent the holiday. Naturally, at some point the conversation centers on what their kids did, with parents taking turns to tell the stories involving their offspring. Thus, one parent starts describing what his kid did, other parents following suit. When his turn comes, Johnny's father utters

(3) Johnny played a silly prank and got some tasty licorice.

In this context, the most natural interpretation of (3) is that, while the relevant perspective for the interpretation of "tasty" is Johnny"s, the perspective relevant for the interpretation of "silly" is not Johnny's (since Johnny himself doesn't think that the prank was silly at all). Instead, the relevant perspective for the interpretation of "silly" could be the father's. This plural reading of (3) is a combination of an autocentric use of a predicate of taste ("silly") and an exocentric use ("tasty"). Another possibility is that the relevant perspective for the interpretation of "silly" is a third person - say, the person who was pranked (assume, for example, that one neighbor has complained earlier about Johnny's prank). This plural reading of (3) is a combination of two exocentric uses of predicates of taste (both "silly" and "tasty" are used by the speaker exocentrically). (3) thus has two plural readings, not only one. ${ }^{4}$

Some readers will no doubt find the plural readings I'm claiming to be available hard to get. One reason for that might be connected with finding it difficult to accept that the relevant perspective for the interpretation of "tasty" in (3) is Johnny's and not the speaker's (i.e., the father's). I acknowledge the difficulty. However, I think the required readings can be made available if we stipulate that the situation is such that i) the father in fact doesn't like licorice

${ }^{4}$ In fact, even more are possible. To give a final example: assume that instead of using "tasty" in (3) exocentrically, the speaker uses it egocentrically; however, "silly" is used exocentrically, with the relevant perspective for its interpretation being a third person (e.g., the neighbor who just complained about the prank). 
and ii) this is known to everyone in the audience. So the father couldn't use "tasty" autocentrically — that would amount to him saying something false. It seems to me that in such a situation (3) is felicitous - in fact, I think that under the stipulations mentioned the plural readings (or at least one of them) are the only possible ones. And such a situation doesn't strike me as incredibly far-fetched -in fact, I believe many real world scenarios are precisely like it.

(3), of course, is not the only example of a sentence containing more than one predicate of taste for which plural readings are available. The following examples, some of them taken from previous work, illustrate the same phenomenon (the first sentences give the context for the ones preceding them, which are the ones that have plural readings):

(4) At this amusement park, we aim to please the whole family. We offer both delicious wines from the local vinery and fun rides in swimming seats - among many other attractions.

(5) Even your vegetarian partner would love the new restaurant. The steaks are of course delicious, but the broccoli burgers are very tasty, too. (Kneer 2015)

(6) We had mixed success with the stuff we bought from the pet store yesterday. The cat food was tasty, but the dog food wasn't. (Second sentence from Anand 2009)

Finally, it is important to stress that the availability of such readings for sentences like (3) and (4)-(6) is not based solely on my own intuitions. In a series of experiments, Kneer (2015) has shown that, for a great number of similar sentences, not only the plural readings are available, but they are the preferred ones in contexts like the ones devised above. When it comes to predicates of taste, then, perspectival plurality is a robust phenomenon. ${ }^{6}$

Perspectival plurality is an interesting phenomenon, but not innocuous. Kneer (2015) and Kneer, Vicente and Zeman (2017) have

\footnotetext{
${ }^{5}$ For many more similar examples, see Kneer 2015, some repeated in Kneer, Vicente and Zeman (2017).

${ }^{6}$ Support for this claim also comes from the fact that predicates of taste are not the only perspectival expressions that allow plural readings, which suggests that the phenomenon is more general. In fact, many of the perspectival expressions mentioned above (footnote 3) have such readings, as well as others not previously mentioned. See Kneer 2015 and Zeman 2018 for discussion.
} 
shown that perspectival plurality creates a problem for (at least certain versions of) relativism. ${ }^{7}$ In particular, the authors mentioned have shown that the phenomenon is especially problematic for Lasersohn's $(2005 ; 2016)$ version of relativism. I won't go into details here, but, in a nutshell, the problem arises for Lasersohn because perspectival plurality is in tension with a principle he takes to be central to his (and, according to him, any) relativist view. Thus, Lasersohn writes: "In a relativist theory, in order to assess a sentence for truth or falsity, one must adopt a stance - that is, truth assessment is always done from a particular perspective" (Lasersohn 2008, p. 326; my emphasis). One might quibble about the interpretation of "a particular perspective", but it seems to me that what Lasersohn means is one and only one perspective. Evidence for this is Lasersohn's treatment of the only example with more than one predicates of taste he considers, and which seems to lead him to generalize to what I will call the "one sentence-one perspective" principle. Thus, Lasersohn focuses on the following sentence (uttered in a situation involving a visit to an amusement park),

(7) Every man gave a woman a fun ride and a tasty dish. ${ }^{8}$ (2008, p. 325),

about which he says that

[i]t can be interpreted at least three ways: The speaker might be expressing his or her own opinion that the rides were fun and the dishes were tasty, or claiming that the each man gave a ride that was fun for him and a dish that was tasty for him, or that each woman received a ride that was fun for her and a dish that was fun for her; but the sentence cannot mean that each man gave some woman a ride that was fun for him, and a dish that was tasty for her. (2008, p. 325; my emphasis.)

What (7) cannot have, according to Lasersohn, is precisely a plural reading. In contrast, the readings that are available, according to Lasersohn, are those in which one and only one perspective is relevant

${ }^{7}$ Other authors have made similar claims - e.g., Cappelen and Hawthorne (2009) and Kissine (2012). However, the examples they use to illustrate perspectival plurality are different, in that (at least some of) the perspectives are made explicit. The examples put forward above show that perspectival plurality arises even if no perspectives are made explicit.

${ }^{8} \mathrm{I}$ am uneasy discussing this example, as it relies heavily on gender stereotypes. However, the example is important because it is the only one of this kind discussed in detail in connection to perspectival plurality. 
for the interpretation of the sentence (call those "singular readings"). It is thus fair to take Lasersohn to mean by "a particular perspective" one and only one perspective. Interpreted in this (reasonable) way, Lasersohn's claim about one of the principles of the relativist framework (amounting to what I called the "one sentence-one perspective" principle) clearly conflicts with perspectival plurality.

One might think that this conflict is not problematic because Lasersohn's rejection of plural readings is circumscribed to a certain type of example (that is, doubly quantified sentences with two predicates of taste) and thus doesn't affect his entire view. But this is a mistake. First, the "one sentence-one perspective" principle that Lasersohn claims is at the heart of his relativist view is entirely general ("in order to assess $a$ sentence"), and thus should apply to the examples provided above. ${ }^{9}$ Second, there are sentences similar to (7) for which plural readings are available. Consider:

(8) Every neighbor let some kids play a silly prank on him and gave them some tasty licorice. ${ }^{10}$

In a context similar with that devised for (3), the most natural interpretation of (8) is that, while the relevant perspectives for the interpretation of "tasty" are those of the kids in the range of the quantifier "some kids", the perspectives relevant for the interpretation of "silly" cannot be theirs. As before, at least two options are available for whose perspective is relevant: that of the speaker and that of the neighbors in the range of the quantifier "every neighbor". So, Lasersohn's ban on plural readings of doubly quantified sentences containing two predicates of taste is not mandated.

Now, there is the question of how widespread commitment to the "one sentence-one perspective" principle is in relativist quarters. To my knowledge, Lasersohn is the only one who adheres to it explicitly. Some critics of relativism (i.e., Kissine (2012)) also think that any relativist position is committed to it. Deciding whether other proponents of relativism also hold it would involve a certain amount of speculation, and this is not the place for doing so. So, while I acknowledge that this is an important issue pertaining to relativism's foundations, I'm happy to concede that perspectival plurality is problematic merely for Lasersohn's position - and, by extension, for any other versions of the view that endorse the aforementioned principle.

\footnotetext{
${ }^{9}$ It is true that Lasersohn doesn't discuss such examples, but considerations of consistency should support the claim that they are targeted by the principle as well.

${ }^{10}$ For many more similar examples, see Kneer 2015, some repeated in Kneer, Vicente and Zeman 2017.
} 
3. Some Possible Relativist Solutions to the Perspectival Plurality Problem

Although examples of sentences with plural readings like (3) and (8) have not been discussed by relativists other than Lasersohn, a number of solutions to the problem raised by perspectival plurality can be proposed on relativism's behalf. In this short section, I will briefly consider three possible ways for the relativist to mitigate the problem. While I will provide some critical remarks to each solution presented, this should not be taken as an attempt to refute them, but rather as an illustration of the theoretical fruitfulness of perspectival plurality.

The first two solutions can be extracted from certain remarks that MacFarlane (2014, chapter 7) makes about treating exocentric and bound uses of predicates of taste. MacFarlane takes on the issue such uses raise for a relativist semantics, and proposes two types of solutions. The first is to claim that predicates of taste have a variable for perspectives in their logical form, but maintain that the value of that variable can sometimes be determined by the context of assessment. More precisely, following Stephenson (2007), MacFarlane appeals to two kinds of variables: regular ones, that get their values from the context of utterance or can be bound, and variables of a special type (what Stephenson calls "PRO ${ }_{J}$ ") that get their values from the context of assessment. The two types of variables correspond to the three uses of predicates of taste mentioned as follows: predicates of taste harbor the regular type of variable when used egocentrically or when bound, but $\mathrm{PRO}_{J}$ when used autocentrically. This helps with perspectival plurality because each predicate of taste in the problematic sentences can harbor any of the two types of variables, and (assuming that the speaker is also the assessor) thus get the two different perspectives needed for the interpretation of plural readings.

MacFarlane's second proposal is that sentences involving predicates of taste used exocentrically or bound in fact express contents that would be literally expressed by making the perspective explicit via predicate-modifying operators like "for $x$ ". To put it concisely, the proposal is to postulate hidden perspective-shifting operators. Such operators have the effect of "creating" perspective-specific contents when predicates of taste are used exocentrically or when bound. However, the idea is that "no syntactic mechanism is posited that gets us this interpretation. Instead, we simply suppose that the speaker expects the hearer to be able to discern that the proposition expressed by [such sentences] is the one she intends to assert" (MacFarlane 
2014, p. 162). When predicates of taste are used autocentrically, though, the contents expressed by the sentences containing them are perspective-neutral. This helps with perspectival plurality because, while the autocentric perspective is provided automatically, the operators provide perspectives other than that of the speaker. We thus get the two different perspectives needed for the interpretation of plural readings.

Ingenuous as the first solution might be, I don't think it amounts to a genuine relativist solution. Although at the outset I conceded that what counts as a "true" form of relativism is partly a matter of stipulation, I take it that postulating at least some contents that are perspective-neutral (and thus, with no variable for perspectives in their logical form) is an essential commitment of a relativist position. This is not the case with the proposal at hand, even if two distinct types of variables are postulated: all the contents expressed by utterances containing predicates of taste are perspective-specific.

MacFarlane's second solution, however, is genuinely relativist (because at least some contents expressed by sentences containing predicates of taste are perspective-neutral). I don't think the following considerations amount to decisive arguments against it, but they should carry some weight. First, let me note that, although not uncommon, postulating hidden structure is to be avoided when alternative options are available. Second, there is some oddity regarding the relation between the syntactic form of a sentence and what, according to MacFarlane, it can express. The problem is not that the hidden structure postulated is missing from the surface, but can be found at the level of syntax; the troublesome part is precisely the opposite claim that it is not to be found there ("no syntactic mechanism is posited that gets us this interpretation"). But, if so, one wonders what mandates the departure from the syntactic configuration of the sentence. True, one of MacFarlane's claims is that syntax and semantics need not go hand in hand. While not much argument is given for this claim, it remains at least a prima facie advantage of a view if it keeps semantics as close as possible to syntax. ${ }^{11}$

Third and finally, one wonders why postulate hidden perspectiveshifters when the effect of shifting the perspective is simply achieved by giving the perspective parameters values that are not those of the speaker/assessor. This is precisely the view held by Lasersohn (2005, 2008), both in the case of exocentric uses and bound ones. MacFar-

\footnotetext{
${ }^{11}$ He also doesn't take these contents to be conveyed pragmatically - the corresponding sentences are "used to assert" (my emphasis) perspective-specific contents.
} 
lane, however, rejects Lasersohn's simpler view. But, as he himself acknowledges $(2014$, p. 156), his arguments against the simpler view are dependent on rejecting Lasersohn's treatment of propositional attitude verbs. Getting into details here would take us very far from the main issues tackled in this paper, but what is rejected is Lasersohn's (2005, 2009) view that "believe" and its ilk are 3-place predicates, taking a subject, a content and a stance as arguments. MacFarlane might be right to do so; however, the point I wish to make is that deciding whether the postulation of hidden perspective-shifting operators is the right way to account for exocentric and bound uses of predicates of taste seems to depend on providing an account of attitude verbs. This seems to me to somehow put the cart before the horse: accounting for simple phenomena like exocentric uses of predicates of taste thus become dependent on accounting for very complex embedding phenomena. ${ }^{12}$

A third way for the relativist to accommodate the phenomenon is to qualify Lasersohn's "one sentence-one perspective" principle by replacing "one sentence" with "one simple sentence", where a simple sentence is one that contains at most one predicate of taste (a view put forward in Kneer, Vicente and Zeman 2017). Complex sentences like (3) are then paraphrased as conjunctions of simple sentences (so that (3), for example, is rendered as "i) Johnny played a silly prank and ii) Johnny got some tasty licorice"). Under such a view, perspectival plurality is accounted for because each simple sentence can in principle be evaluated with respect to a different perspective. But although such a "paraphrasing strategy" goes a long way to address the problem, it is not entirely without flaws. First, despite the significant number of putatively problematic examples addressed in the paper mentioned, it is doubtful that all possible complex sentences with plural readings are paraphrasable in the way suggested. Second, and more importantly, the strategy seems to lack syntactic credentials: it is very unlikely that from the point of view of contemporary syntax complex sentences (especially those involving quantifiers) should be broken down syntactically into simple ones. One consideration that

${ }^{12}$ A different solution would be to claim that predicates of taste are ambiguous between 1-place predicates and 2-place predicates, with the additional proviso that they are 1-place predicates only when used autocentrically and 2-place predicates when used egocentrically or when bound. This would help with the perspectival plurality problem because there won't be any need for the second perspective to be provided by the index since it is already part of the logical form of the relevant sentences as a result of filling in the variable(s). While possible, such a view strikes me as completely ad-hoc. 
could mitigate this objection is that the decomposition is made for purposes of evaluation only, and thus the level of representation at which the paraphrase is given is independent of syntax. Assuming a tight overlap between the syntactic and the semantic level, postulating this level of representation amounts to an additional burden - a burden that, all things being equal, is best avoided. ${ }^{13}$

To sum up, none of the three strategies presented is optimal: the first doesn't amount to a genuine relativist view, the second depends on the analysis of complex embedding phenomena and the third is in tension with contemporary syntax or too costly. Thus, if there are less problematic and more natural solutions on the table, they should be preferred. To be sure, the remarks above are far from showing that the views envisaged are doomed, and no doubt they merit closer investigation. But while I acknowledge the potential for these solutions to be worked out, I think exploring a different option might be beneficial for the debate. This is precisely what I set out to do in the reminder of the paper. ${ }^{14}$

\section{Multiple Indexing Relativism: A Sketch}

As we have seen in section 1, relativism's main feature is that it introduces a parameter for perspectives in the index. Perspectival plurality raises the doubt that one such parameter is enough. A natural move by the relativist would be, then, to postulate more than one parameter for perspectives. This is an instance of a more general strategy known as "multiple indexing". ${ }^{15}$

In the way I'm using the term in this paper, "multiple indexing" refers to the introduction of more than one parameter of the same

${ }^{13}$ See Kneer 2015 for other objections to this strategy.

${ }^{14}$ Needless to say, yet other solutions might be proposed. For example, one might be to adopt a relativist-friendly dynamic framework. I have discussed only static solutions in this section because i) the great majority of participants in the debate over semantic content use the static Kaplanian framework I also assumed at the outset; ii) as far as I know, relativism has not been so far couched in a dynamic framework.

${ }^{15}$ Authors like Kamp (1971), Vlach (1973), Lewis (1980), Kaplan (1989) and, more recently, Rey (2016), among many others, appeal to what is known as "double indexing": the postulation of two parameters of the same kind in the semantic apparatus. Of course, there are great differences between the authors mentioned, the most important ones stemming from the role each of them gives to the two parameters and resulting in radically different views on the contents of utterances (for a recent paper on these differences, see Rabern and Ball 2017). The view proposed in what follows, although related to the double indexing strategy, is different in several respects that will become clear below. 
kind in the index. More precisely, the proposal is that each utterance is to be evaluated with respect to sequences of parameters of a certain kind. The essential feature of a sequence is that it provides an ordering on its elements (in this case, parameters) that are systematically related to the expressions to which they correspond. Thus, the value of the first parameter in the sequence is used to evaluate the first (occurrence of the) expression it corresponds to, the value of the second parameter in the sequence is used to evaluate the second (occurrence of the) expression it corresponds to and so on. Multiple indexing is thus a wholesale, global strategy to assess for truth complex sentences by (locally) providing values of the parameters used for the evaluation of each (occurrence of the) expression they correspond to.

In the case of predicates of taste, the sequence I propose to introduce is composed of parameters for perspectives, with the result that each parameter will provide a value for the perspective used to evaluate each (occurrence of the) predicate of taste it corresponds to. ${ }^{16}$ The strategy helps with perspectival plurality because it allows that, in principle, each (occurrence of a) predicate of taste can be evaluated with respect to a different perspective. This is exactly what cannot happen if one upholds the principle considered by Lasersohn to be at relativism's roots. But it also has no problem with what I called singular readings - for example, those that Lasersohn claimed are the only ones available in the case of (7). What happens in such cases is that the values of all the parameters for perspectives in the sequence are the same - that is, all the predicates of taste are evaluated with respect to the same value, and, thus, the sentence itself.

Let me illustrate how the strategy is applied, first by giving the truth conditions of (some of) the readings of the problematic sentences examined above. Taken in itself, without being uttered in a specific context - that is, before supplying any contextual information relevant for the truth evaluation of an utterance of (3), including the values for the two parameters for perspectives postulated - the template for its truth conditions looks like this:

(9) [[Johnny played a silly ${ }^{1}$ prank and got some tasty ${ }^{2}$ licorice] $]^{c, w,<p 1, p 2>}=1$ iff Johnny played a silly prank in $w$ according to the value of $p l$ and got some tasty licorice in $w$ according to the value of $p 2$,

\footnotetext{
${ }^{16}$ The correspondence will be formally implemented by superscripting each occurrence of a predicate of taste and indexing it to the position of each parameter in the sequence. See below.
} 
where $p 1$ and $p 2$ are the two parameters for perspectives in the sequence introduced, the superscripts on the two predicates of taste represent the order in which they appear and the co-indexing of the parameters with those superscripts signifies that they correspond to the predicates superscripted ( $p n$ corresponds to $\Phi^{n}$, where $\Phi$ is a predicate). ${ }^{17}$ Now, when giving the truth conditions for utterances of (3) in specific contexts - that is, when contextual information relevant for their truth evaluation is supplied, including the values for the two parameters $p 1$ and $p 2$ - we in fact give truth conditions for the sentence's various readings. Thus, consider first one of the plural readings of (3) made salient in section 2, when uttered in the Halloween context - say, the one according to which the relevant perspective for the interpretation of "tasty" is Johnny's, while the relevant perspective for the interpretation of "silly" is the speaker's. This plural reading of (3) is represented as follows:

(10) [[Johnny played a silly ${ }^{1}$ prank and got some tasty ${ }^{2}$ licorice $]]^{c, w,<p 1[\text { speaker }], p 2[\text { Johnny }]>}=1$ iff Johnny played a silly prank in $w$ according to the speaker's perspective and got some tasty licorice in $w$ according to Johnny's perspective,

where $p n[v]$ should be read as " $v$ 's perspective is the value of the $p n$-th parameter for perspectives" — so that the value of $p$ I [speaker] is the speaker's perspective and the value of $p 2$ [Johnny] is Johnny's perspective. Next, consider one of (3)'s singular readings, as uttered in a different context - say, the one according to which the relevant perspective for interpreting both predicates of taste is the speaker's. This singular reading is represented as follows:

(11) [[Johnny played a silly ${ }^{1}$ prank and got some tasty ${ }^{2}$ licorice $]]^{c, w,<p 1[\text { speaker }], p 2[\text { speaker }]>}=1$ iff Johnny played a silly prank in $w$ according to the speaker's perspective and got some tasty licorice in $w$ according to the speaker's perspective.

${ }^{17}$ As it stands, (9) is silent about which context is it that provides the values for the two perspective parameters. Thus, depending on the choice one makes in that respect, there are two possible versions of Multiple Indexing Relativism: a moderate one and a radical one. The general view is thus compatible with various post-semantic takes on the provision of values for the perspective parameters in the index. It is true that in discussing the various examples of plural readings I only made reference to the context of utterance. This, however, shouldn't diminish their importance. It is an interesting question to explore whether the phenomenon illustrated appears when the context that provides the values for the perspectives parameters is the context of assessment (my hunch is yes). 
The difference in readings is thus accounted for by giving different values to the perspectives in the sequence. The framework is flexible enough to capture all the possible plural readings (see section 2), but also all the possible singular readings of sentences like (3). ${ }^{18}$

As for multi-perspectival readings of complex sentences with quantifiers like (8), I think there is more than one way to go, depending on the account of binding adopted. For example, we can preserve the spirit of Lasersohn's (2008) account of binding — "index binding"but modify its letter so as to allow multiple indexing in the picture. ${ }^{19}$ In essence, index binding replaces binding in the object language with binding in the meta-language. According to the proposal, quantifiers can bind both variables in the object language and variables in the meta-language. For example, in the sentence "Everyone got something tasty" the quantifier "everyone" binds both a variable in the object language and one in the meta-language: the first is the subject variable of the verb "get", while the second is the variable for perspectives. Combined with the "one sentence-one perspective" principle endorsed by Lasersohn, index binding has the consequence that all the predicates in a sentence like (8) have to be bound by one quantifier at most. However, as we have seen, due to perspectival plurality, the "one sentence-one perspective" has to be dropped. What this means for index binding is that we need to allow the possibility that each predicate of taste in a sentence like (8) is bound by a different quantifier (just like in object language variable binding). Given that binding a predicate of taste is done via binding the parameter for perspectives in the sequence to which it corresponds, we need to allow the possibility that a quantifier binds only the $n$-th parameter for perspectives in the sequence - that corresponding to the $n$-th predicate in the sentence - instead of binding all the parameters for perspectives in the sequence.

Let me illustrate how the account applies concretely, again by giving the truth conditions of (some of) the readings of (8). Taken in itself, without being uttered in a specific context - that is, before sup-

${ }^{18}$ An important question that can be asked at this point is how exactly are the values of the parameters for perspectives determined in a certain context. I cannot provide a detailed answer here, but the short one is: at least partially, by the intentions of the speaker.

${ }^{19}$ Index binding has been recently criticized by Snyder (2013). I ignore his criticisms in what follows, not because they are not important, but because I want to focus on showing how index binding can be implemented in a Multiple Indexing Relativist framework. For an alternative way to handle binding in a relativist-friendly manner, using variadic operators, see Zeman (2015). 
plying any contextual information relevant for the truth evaluation of an utterance of (8), including the values for the two parameters for perspectives postulated - the template for its truth conditions (simplifying greatly) looks like this:

(12) [[Every neighbor let some kids play a silly ${ }^{1}$ prank on him and gave them some tasty ${ }^{2}$ licorice] $]^{c, w,<p 1, p 2>}=1$ iff Every neighbor $x$ let some kid $y$ play a silly prank on $x$ in $w$ according to the value of $p l$ and $x$ gave $y$ some tasty licorice in $w$ according to the value of $p 2$.

As in the case of (3), when giving the truth conditions for utterances of (8) in specific contexts, we in fact give truth conditions for the sentence's various readings. Thus, consider first one plural reading of (8) made salient in section 2, when uttered in the Halloween context - say, one in which the relevant perspectives for the interpretation of "tasty" are those of the kids in the range of the quantifier "some kids", while the relevant perspectives for the interpretation of "silly" are those of the neighbors in the range of the quantifier "every neighbor". This reading is represented as follows:

(13) [[Every neighbor let some kids play a silly ${ }^{1}$ prank on him and gave them some tasty $^{2}$ licorice $\left.]\right]^{c, w,<p l[x], p 2[y]>}=1$ iff Every neighbor $x$ let some kid $y$ play a silly prank on $x$ in $w$ according to $x$ 's perspective and $x$ gave $y$ some tasty licorice in $w$ according to $y$ 's perspective,

where "every neighbor" and "some kids" quantify both over object language variables and over parameters for perspectives in the index, as Lasersohn's index binding requires, with each of them quantifying over one of the parameters in the sequence (the former over $p 1$, the latter over $p 2$ ). Next, consider two singular readings of (8), as uttered in different contexts - say, one in which the relevant perspective for the interpretation of both predicates of taste is the speaker's, the other in which the relevant perspectives for the interpretation of both predicates of taste are those of the neighbors in the range of the quantifier "every neighbor". The two singular readings are represented by (14) and (15), respectively:

(14) [[Every neighbor let some kids play a silly ${ }^{1}$ prank on him and gave them some tasty ${ }^{2}$ licorice $\left.]\right]^{c, w,<p 1[\text { speaker }], p 2[\text { speaker }]>}=1$ iff Every neighbor $x$ let some kid $y$ play a silly prank on $x$ in $w$ according to the speaker's perspective and $x$ gave $y$ some tasty licorice in $w$ according to the speaker's perspective. 
(15) [[Every neighbor let some kids play a silly ${ }^{1}$ prank on him and gave them some tasty ${ }^{2}$ licorice $\left.]\right]^{c, w,<p 1[x], p[x]>}=1$ iff Every neighbor $x$ let some kid $y$ play a silly prank on $x$ in $w$ according to $x$ 's perspective and $x$ gave $y$ some tasty licorice in $w$ according to $x$ 's perspective.

The difference in readings is thus accounted for by giving different values to the perspectives in the sequence. The framework is flexible enough to capture all the possible plural readings (see section 2), but also all the possible singular readings of sentences like (8).

\section{Objections and Replies}

In this last section, I address three possible objections to Multiple Indexing Relativism: the ad-hocness objection, the complexity objection, and the objection from unavailable readings. The answers to these objections will hopefully give a more complex picture of the view on offer.

\subsection{The Ad-Hocness Objection}

A fair objection to the strategy employed above in reply to the perspectival plurality problem is that it is ad-hoc. While the solution might be formally adequate, one might feel that appeal to sequences of parameters instead of a single parameter is an unmotivated (besides the need to solve the perspectival plurality problem, that is) departure from the traditional Kaplanian framework.

Yet, even a quick look at the literature on multiple indexing is enough to dispel this impression. As I already mentioned, similar views have been put forward in relation to other expressions and for solving a wide range of philosophical problems. For example, Cresswell's $(1973,1990)$ postulation of multiple parameters for possible worlds is meant to account for complex sentences with modal operators; Vlach's (1973) system with multiple time parameters was designed to account for complex phenomena like sequence of tense; more recently, Rabern (2012) has argued that a framework with multiple indices is needed to deal properly with quantification in Kaplan's system, etc. Multiple indexing thus seems to be a widespread tool in dealing with linguistic phenomena involving a wide range of expressions. Application to predicates of taste and other perspectival expressions is thus a natural move. ${ }^{20}$

${ }^{20}$ In addition, it can be argued that the form of multiple indexing adopted here 
As for solving philosophical problems, I will give here only one example. In the literature on belief, accounting for the special character of thinking about oneself has had a central place. One way to account for this special character is to postulate a special type of belief —namely, the so-called de se belief (see, e.g., Lewis 1979), which is taken to have a certain type of content: a de se, or "centered" content. In a nutshell, a centered content has to be evaluated with respect to a center (a tuple of parameters containing a possible world, a time and an individual, so that the individual is located in that world at that time). However, while centered contents have been taken to adequately capture the special character of thinking about oneself, ${ }^{21}$ it is widely acknowledged (Stalnaker 1981; Torre 2010; Ninan 2010; Stojanovic 2012; Weber 2013) that communicating thoughts about oneself is problematic. For example, if the belief one would express using the true sentence

\section{(16) I'm hungry}

involves a centered content with the speaker as the center, communicating this belief to others would be problematic, as the content would be false when centered on the hearer.

A recently proposed solution to this problem consists in construing the contents of beliefs expressed by sentences like (16) as multicentered - that is, as contents that are to be evaluated with respect to a sequence of centers (Torre 2010; Ninan 2010; Kindermann 2019). The communication problem is solved by introducing a sequence with two centers only - one tracking the speaker, the other the hearer, but the framework can easily be modified to contain more centers if needed. Technically, this simply means introducing a sequence of two parameters for individuals in the index - that is, a multiple indexing strategy in my sense. The details of how to best understand multi-centered contents need not detain us here; ${ }^{22}$ the

is not so dramatic a departure from the Kaplanian view framework as one might think. See Zeman 2018.

${ }^{21}$ Not by everyone, of course. See, for example, Stalnaker 1981. For a more radical view that denies the special character of such thinking, see Cappelen and Dever 2014.

22 Thus, Ninan takes the pair of centers to be a sequence that imposes an order on the interlocutors in a certain context, the first center remaining fixed on one of the interlocutor, the second on the other, while for Torre the order of the centers is arbitrary. For both views, however, the truth conditions of (16) are represented as $[[\mathrm{I} \text { am hungry }]]^{c, w,\langle x, y\rangle}=1$ iff there is a $y$ such that $y$ is $x$ 's addressee in $w$ and $x$ is hungry in $w$. 
point is that multi-centering is an example of successfully employing a multiple indexing strategy to solve a pressing problem. While (16) is different from (3), in that it contains only one problematic expressions, not two, the solution is structurally very close to Multiple Indexing Relativism. It is hard to consider a view ad-hoc if it solves more than one important problem.

\subsection{The Complexity Objection}

In addressing the recent advent of relativism, ${ }^{23}$ Glanzberg (2007) worries that its widespread adoption would lead to a view that is too complex, which in turn leads to problems with semantic competence. In a nutshell, the worry is that relativism, by introducing a wide range of parameters in the index, casts doubt about the possibility of learning and computing languages. Obviously, these worries are augmented if one adopts Multiple Indexing Relativism, which is more complex than the canonical versions of relativism found in the literature. It is not only one parameter of a certain kind that we need to worry about now, but an entire sequence.

To make things even more complicated, there is another aspect of the theory put forward here that needs to be considered. In representing the truth conditions of sentences like (3) and (8), I have used a sequence of parameters with only two perspectives for convenience. But given the existence - at least in principle - of sentences with an infinite number of predicates of taste, and the possibility of plural readings of such sentences, the sequences I propose to introduce will have to be infinite as well. ${ }^{24}$ The worry that the picture proposed is too complex is thus even more pressing.

One (very blunt) way to respond to this worry is to simply bite the bullet and claim that since our language is so complex, we need

${ }^{23}$ Glanzberg's primary target is Richard's (2004) view. According to Richard, relativism should be applied to all expressions for which there is "accommodation and negotiation". As Glanzberg is aware, this is not other authors' main reason for adopting relativism. I haven't considered Richard's version of relativism and his motivations in this paper, but the critical points Glanzberg makes transfer to other versions as well.

${ }^{24}$ Is the formal implementation of such a theory possible? Vlach (1973), as well as Cresswell (1990), discuss the possibility of adopting systems with infinite indexing and find it formally unproblematic. Vlach (1973, Appendix) puts forward a view that employs a finite but non-limited sequence of perspective parameters. Rabern's 2012 makes a stronger claim, namely that a non-finite number of parameters is in fact necessary for semantics. Thus, from a purely formal point of view, there is no obstacle in using sequences that are infinite or finite but non-limited. See the formal system given in the Appendix for an example. 
correspondingly complex tools to handle it. ${ }^{25}$ Yet, this answer doesn't quite cut it: there is something unsettling about having to compute and learn expressions whose truth depends on possibly infinite sequences of a whole range of parameters. However, I don't think the relativist should be alarmed. If I understand Glanzberg's objection properly, the worry pertains to an all-encompassing version of relativism meant to be applied to a great number of natural language expressions. If that is the case, we will indeed end up with a great number of types of parameters in the index. However, relativism need not be global: while the relativist might wish to apply the theory to many types of expressions, it might turn out that for some of them a different treatment is more adequate. Whether this is so or not depends on data that I haven't investigated in this paper. This already imposes a limit on the number and kinds of parameters postulated. A further limitation comes from the fact that many expressions can be subsumed to the same parameter: for example, what I called in this paper "perspectival expressions" can all be subsumed under perspectives. Again, whether this can be achieved depends on data that I haven't addressed. The same considerations apply mutatis mutandis to Multiple Indexing Relativism: introducing sequences of parameters of a certain kind is compatible with abstaining from introducing sequences of parameters of other kinds, while subsuming several types of expressions under the same sequence of parameters (if the data mandate it) is certainly a possible way to construct the theory. As for infinite sequences, there is hope here too: although contents will be relative to such sequences, most of the parameters will be idle, since in fact sentences with a great number of predicates of taste will most likely never be uttered. (The idleness of perspectives is a claim already familiar to the relativist, given that there are sentences that don't contain any predicates of taste, yet the perspectives are present in the semantic apparatus — see, e.g., Kölbel 2009.) There is an alternative route, too: instead of taking contents to be functions from sequences that are infinite or finite but non-limited to truth values, one can see them as partial functions from sequences with a number of parameters $n$ to truth values. The partial functions are obtained by imposing the condition that $n$ is equal to the number of predicates of taste in the sentence whose semantic value is evaluated for truth. So, the burden that relativism might put on computability

${ }^{25}$ As I already mentioned in the previous footnote, Vlach (1973), Cresswell (1990) or Rabern (2012) are great examples both of illustrating how complex natural language can be and of appealing to non-finite parameters in semantics. 
and learnability that Glanzberg warns us against is not that heavy, after all.

Another way in which the relativist can reply to the complexity worry is to go instrumentalist: that is, to refrain from investing the theory with psychological reality. Understanding the relativist theory, either in its canonical or in the Multiple Indexing version, merely as model of linguistic phenomena simply makes the objection addressed misfire. But even if the relativist would claim psychological reality for her theory, the objection needs to be backed up by a significant amount of empirical data - data that, I dare to claim, we don't possess at the moment. ${ }^{26}$ In the absence of such data, the objection from complexity, I claim, should not be taken too seriously.

\subsection{The Objection from Unavailable Readings}

The last, and very important, possible objection to Multiple Indexing Relativism that I'm considering has to do with its empirical adequacy. As we have seen in section 2, Lasersohn claims that in the case of (7) no plural readings are available, and that claim has presumably led him to adopt the "one sentence-one perspective" principle. The data presented there clearly show that the principle is false. However, it could still be the case that for certain sentences no plural readings are available in any context (perhaps (7) is such a case). The objection is that, by not ruling out the possibility of such readings, Multiple Indexing Relativism gets the data wrong.

This is a serious concern that merits a much more developed answer than I'm able to offer here. Here are, however, two ways to

\footnotetext{
${ }^{26}$ There is some experimental work on the acquisition of perspectives in connection with perspectival expressions. Thus, Roeper (2016) (citing work done in collaboration with Chloe $\mathrm{Gu}$ ) has found that children assume by default a "general point of view" (which, supposedly, is not relativized to perspectives) when they learn such expressions. The authors think that this conflicts with relativism about the expressions in question. However, I have doubts that the existence of a general point of view is incompatible with relativism. First, even if it is true that when children learn perspectival expressions they assume a general point of view, the question remains open what happens when they get exposed to different perspectives. That is, the adoption of a general point of view doesn't settle the matter of how to treat such expressions as used by adult individuals. Moreover, while the adoption of a general point of view by children might be evidence for absolutism or invariantism, it is evidence against not only relativism, but against any view that accepts that perspectival expressions give raise to the kind of context-sensitivity illustrated in section l. Finally, the studies carried out don't tackle plural readings, so we are still very much in the dark about what actually happens when children learn sentences like (3).
} 
address the issue. First, it might be argued that, in certain intricate contexts, sentence (7) does get a plural reading. Lasersohn excludes all plural readings of the sentence, and he insists on the one according to which the rides were fun for the men and the dishes were tasty for the women. However, the readings in which one of the predicates is used autocentrically by the speaker or from the point of view of another person are also plural readings. Such readings seem to be available. For example, imagine that several pairs of man and women enter a competition in an amusement park about how much fun they had together. As it turns out, all the pairs did was to go on a ride and have a meal. Suppose further that they all went on the same ride and had the same dish. However, that while everyone liked the ride, no one liked the dish - except the moderator of the competition. Wanting to make their preference known, the moderator utters (7), to the dismay of the participants. Or (perhaps more realistically), they utter (7) because they were paid by the restaurant to do so. It seems to me that (7) can be used by the moderator in both situations, with the sentence getting two different plural readings.

As for the plural reading that Lasersohn focuses on, it also seems to be available. In the amusement park competition scenario above, suppose that none of the women liked the ride(s), but all men did, and that none of the men liked the dish(es), but all women did. Suppose that what matters towards winning the competition is that at least one member of each pair had a pleasurable experience. The moderator can, it seems to me, describe the situation by uttering (7) (perhaps leading to a draw) — case in which the sentence has the plural reading that Lasersohn unequivocally excludes. ${ }^{27}$

But even assuming that plural readings of (7) are not available, the Multiple Indexing Relativist is not completely at a loss. For what is certainly true is that the view proposed fares at least as well as other relativist positions. Compare, first, Multiple Indexing Relativism with the canonical version of the view. Consider two ways in which recalcitrant cases are dealt with in semantics. On one

${ }^{27}$ A referee suggests an example structurally very similar to (7), about which the referee claims to access a plural reading: "Every man took a woman on a thrilling rollercoaster and then to a fantastic romantic comedy." The referee maintains that the plural reading is more easily available by playing into gender stereotypes. I agree with both of the referee's points. As I mentioned in footnote 8, I am uneasy discussing examples like (7) precisely for that reason; my attempts to come up with scenarios in which (7) has a plural reading might thus add insult to injury. However, doing so serves an important dialectical purpose: to show that Lasersohn is mistaken and to fend off the objection from unavailable readings. 
hand, a theory might be too restrictive: it might rule out readings that are, in fact, available. On the other hand, a theory might be too permissive: it might allow readings that, in fact, are not to be found. The canonical relativist position seems to belong to the former category; Multiple Indexing Relativism to the latter.

Which of the two mistakes is more palatable? It seems to me that, generally speaking, it is better to err on the permissibility side than on the other. However, providing arguments for each side would necessitate going into complex methodological issues that I cannot take up here. A reasonable position is that neither of the two ways of erring is preferable to the other. If this is the case, then, it remains true that Multiple Indexing Relativism doesn't fare worse than the canonical version of the view. Additionally, and perhaps more importantly in this context, the Multiple Indexing Relativist also doesn't fare worse when compared to the other ways of solving the perspectival plurality problem briefly presented in section 3 . For all that has been said there, neither of the two solutions to the perspectival plurality problem extracted from MacFarlane's (2014) remarks nor the "paraphrasing strategy" of Kneer, Vicente and Zeman (2017) give us the constraints we need to exclude the unwanted plural readings. ${ }^{28}$

This second way of addressing the issue might not sound very promising, in that it leaves the issue of the constraints on available readings open. I acknowledge that this is one of the pressing problems Multiple Indexing Relativism has not yet a definite answer to. I hope, however, that the view is attractive enough to mandate future consideration. ${ }^{29}$

\section{Appendix}

To put more flesh on the bones of the Multiple Indexing Relativist framework, in this appendix I will give a semantics for a very small

${ }^{28}$ I thank a referee for the suggestion of making this comparison.

${ }^{29}$ Another possible answer would appeal to pragmatic principles in order to exclude the unwanted readings. A referee is rightly skeptical about the efficiency of such a strategy. First, pragmatic principles could be appealed to by any view in the debate and so, in principle, any view could be made to work (whether this is so depends, of course, on the specific principles proposed). Second, by employing such principles one might overdo it by excluding "plural" readings of sentences similar to (7) but without predicates of taste; "Every adult gave a child their food but was not thanked by them" is the example given by a referee. This being said, I do think that some kind of pragmatic principle might be needed to rule out plural readings of sentences like "Licorice is tasty, but it's not", which sound bad (perhaps something along the lines of "don't change perspectives mid-sentence unless it is necessary"). I leave investigation of this issue for future research. 
portion of language, containing only two common nouns ("prank" and "licorice"), a proper name ("Johnny"), two predicates of taste ("silly" and "tasty"), two verbs ("played" and "got") and two quantifiers ("a" and "some"). I begin with the definition of the semantic types used:

a) e, $t$ are semantic types.

b) If $\sigma$ and $\tau$ are semantic types, then $\langle\sigma, \tau\rangle$ is a semantic type.

c) Nothing else is a semantic type.

The semantic denotation domains associated with these types are defined as follows:

d) $\mathrm{D}_{e}$ is the set of individuals;

e) $\mathrm{D}_{t}$ is the set of truth values $(\{0,1\})$;

f) For any semantic type $\sigma$ and $\tau, \mathrm{D}_{<\sigma, \tau>}$ is the set of all functions from $\mathrm{D}_{\sigma}$ to $\mathrm{D}_{\tau}$.

Other symbols used:

'f', 'g', 'h', 'i' symbolize variables for functions;

'x', 'y', 'z', 'm', 'n', 'o', 'r', 's', 'a', 'b', 'd', 'e' symbolize variables for individuals;

' $\mathrm{C}$ ' stands for the set of contexts;

' $\mathrm{W}$ ' stands for the set of possible worlds;

' $\mathrm{P}$ ' stands for the set of sequences of perspectives.

The lexical entries for the expressions in the fragment are as follows: $\left[\left[\text { silly }^{i}\right]\right]^{c, w,<p l, \ldots p i, \ldots p n>}=\lambda x \in D_{e} . x$ is silly in $w$ according to perspective $p i$.

$\left[\right.$ tasty $\left.\left.^{i}\right]\right]^{c, w,<p 1, \ldots p i, \ldots p n>}=\lambda x \in D_{e} . x$ is tasty in $w$ according to perspective $p i$.

$[[\mathrm{Johnny}]]^{c, w,<p 1, \ldots p i, \ldots p n>}=$ Johnny.

$[[\operatorname{prank}]]^{c, w,<p l, \ldots p i, \ldots p n>}=\lambda x \in D_{e} . x$ is a prank in $w$. 
[[licorice] $]^{c, w,<p 1, \ldots p i, \ldots p n>}=\lambda x \in D_{e} . x$ belongs to the kind licorice in $w .^{30}$

[[played] ] $]^{c, w,<p l, \ldots p i, \ldots p n>}=\lambda x \in D_{e} . \lambda y \in D_{e} . y$ played $x$ in $w$. [[got] $]^{c, w,<p l, \ldots p i, \ldots p n>}=\lambda x \in D_{e} . \lambda y \in D_{e} . y$ got $x$ in $w{ }^{31}$

$[[\mathrm{a}]]^{c, w,<p l, \ldots p i, \ldots p n>}=[[\text { some }]]^{c, w,<p l, \ldots p i, \ldots p n>}=\lambda f \in D_{<e, t>}$. there is an $x \in D_{e}, f(x)=1 .^{32}$

$[[\text { and }]]^{c, w,<p l, \ldots p i, \ldots p n>}=\lambda f \in D_{<e, t>} . \lambda g \in D_{<e, t>} . \lambda x \in D_{e}$. $f(x)=1$ and $g(x)=1 .^{33}$

As can be seen on this list, some expressions are perspectival - that is, their interpretation depends on a perspective ("silly" and "tasty"), others are not (all the others). In the case of the latter, the parameters in the sequence are idle. All the perspectival expressions are indexed - that is, given numbers as superscripts that correspond to the position of the perspective parameter in the sequence which is responsible for their interpretation. The number of perspectival expressions in a sentence thus determines the number of active perspectives in the sequence.

In order to compute the truth conditions of sentences like (3), the following two combination rules are needed (taken from Heim and Krazer (1998) and suitably modified):

Functional Application: If $\alpha$ is an expression composed of two expressions $\beta$ and $\gamma$, then, for any $c \in C, w \in W$ and $<p l, \ldots p i, \ldots p n>\in P$, if $[[\beta]]^{c, w,<p l, \ldots p i, \ldots p n>}$ is a function whose domain contains $[[\gamma]]^{c, w},<p 1, \ldots p i, \ldots p n>$, then $[[\alpha]]^{c, w}$, $<p 1, \ldots p i, \ldots p n>=\left[[[\beta]]^{c, w,<p l, \ldots p i, \ldots p n>}\right]\left([[\gamma]]^{c, w,<p l, \ldots p i, \ldots p n>}\right)$, where " $[\phi](\psi)$ " symbolizes the application of function $\phi$ to argument $\psi$.

Predicate Modification: If $\alpha$ is an expression composed of two expressions $\beta$ and $\gamma$, then, for any $c \in C, w \in W$ and $<p l, \ldots p i, \ldots p n>\in P$, if $[[\beta]]^{c, w,<p l, \ldots p i, \ldots p n>}$ and $[[\gamma]]^{c, w}$, $<p 1, \ldots p i, \ldots p n>$ are both functions of type $\left\langle e, t>\right.$, then $[[\alpha]]^{c, w}$,

${ }^{30}$ I ignore complications with mass nouns that appear here.

${ }^{31}$ I'm ignoring tense for both "played" and "got".

${ }^{32}$ Here, as in the paper as a whole, I'm ignoring assignment functions needed for the semantic treatment of quantifiers. They will have to be introduced in giving the semantics for sentences like (8), for example.

33 "And" is treated as a verb phrase conjunction, not a sentential one. 


$$
\begin{aligned}
& <p 1, \ldots p i, \ldots p n>=\lambda x \in D_{e} .\left[[[\beta]]^{c, w,<p l, \ldots p i, \ldots p n>}\right](x)=\left[[[\gamma]]^{c, w},\right. \\
& <p 1, \ldots p i, \ldots p n>](x)=1 .
\end{aligned}
$$

With these tools in hand, the truth conditions of (3) are computed as follows:

$\left[\left[\mathrm{Johnn} \text { played a silly }{ }^{\mathrm{l}} \text { prank and got some tasty }{ }^{2} \text { licorice }\right]\right]^{c, w}$, $<p 1, p 2, \ldots p n>=\left[\right.$ [[played a silly ${ }^{1}$ prank and got some tasty ${ }^{2}$ licorice $\left.]]^{c, w,<p 1, p 2, \ldots p n>}\right]\left([[\mathrm{J} \text { ohnny }]]^{c, w},<p 1, p 2, \ldots p n>\right)=[$ [ [ [[and $\left.]\right]$ $c, w,<p 1, p 2, \ldots p n>]$ ([[got some tasty ${ }^{2}$ licorice $\left.\left.\left.]\right]^{c, w,<p l, p 2, \ldots p n>}\right)\right]$

([[played a silly ${ }^{1}$ prank $\left.\left.\left.]\right]^{c, w,<p l, p 2, \ldots p n>}\right)\right]\left([[\mathrm{Johnny}]]^{c, w,<p l, p 2,}\right.$ $\ldots p n>$ ).

The two complex verb phrases are computed as follows:

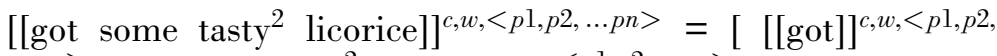
$\ldots p n>]$ ([[some tasty ${ }^{2}$ licorice $\left.\left.]\right]^{c, w,<p 1, p 2, \ldots p n>}\right)=\left[[[\text { got }]]^{c, w}\right.$, $<p 1, p 2, \ldots p n>] \quad\left(\left[[[\text { some }]]^{c, w},<p 1, p 2, \ldots p n>\right] \quad\left(\left(\left[\left[\text { tasty }^{2}\right]\right]^{c, w,<p l,}\right.\right.\right.$ $\left.\left.\left.p 2, \ldots p n>)\left([[\text { licorice }]]^{c, w},<p 1, p 2, \ldots p n>\right)\right)\right]\right)=\left[\lambda x \in D_{e} . \lambda y \in D_{e}\right.$. $y$ got $x$ in $w]\left(\left[\lambda f \in D_{<e, t>}\right.\right.$. there is a $\left.z \in D_{e}, f(z)=1\right]$ $\left(\left(\lambda m \in D_{e} . m\right.\right.$ is tasty in $w$ according to perspective $\left.p 2\right)$ $\left(\lambda n \in D_{e} . n\right.$ belongs to the kind licorice in $\left.\left.\left.w\right)\right)\right)=\left[\lambda x \in D_{e}\right.$. $\lambda y \in D_{e} . y$ got $x$ in $\left.w\right]\left(\left[\lambda f \in D_{<e, t>}\right.\right.$. there is a $z \in D_{e}, f(z)$ $=1]\left(\lambda o \in D_{e} . o\right.$ is tasty in $w$ according to perspective $p 2$ and belongs to the kind licorice in $w))=\left[\lambda x \in D_{e} . \lambda y \in D_{e} . y\right.$ got $x$ in $w$ ] (there is a $z \in D_{e}$ such that $z$ is tasty in $w$ according to perspective $p 2$ and belongs to the kind licorice in $w)=\lambda y \in D_{e}$. $y$ got a thing that is tasty in $w$ according to perspective $p 2$ and belongs to the kind licorice in $w$.

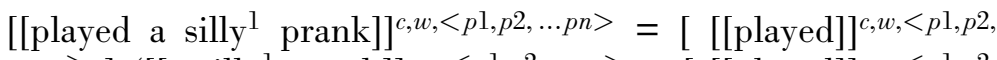
$\ldots p n>]\left(\left[\left[\text { a silly }^{1} \text { prank }^{c}\right]^{c, w,<p 1, p 2, \ldots p n>}=[\text { [[played] }]^{c, w,<p l, p 2,}\right.\right.$ $\ldots p n>]\left(\left[[[\mathrm{a}]]^{c, w,<p 1, p 2, \ldots p n>}\right]\left(\left(\left[\left[\mathrm{silly}^{\mathrm{l}}\right]\right]^{c, w,<p 1, p 2, \ldots p n>}\right)([[\mathrm{prank}]]\right.\right.$ $c, w,<p 1, p 2, \ldots p n>))])=\left[\lambda a \in D_{e} . \lambda b \in D_{e} . b\right.$ played $a$ in $\left.w\right]$ $\left(\left[\lambda g \in D_{<e, t>}\right.\right.$. there is an $\left.s \in D_{e}, g(s)=1\right]\left(\left(\lambda d \in D_{e} . d\right.\right.$ is silly in $w$ according to perspective $p l)\left(\lambda e \in D_{e}\right.$. e is a prank in $w)))=\left[\lambda a \in D_{e} . \lambda b \in D_{e} . b\right.$ played $a$ in $\left.w\right]\left(\left[\lambda g \in D_{<e, t>}\right.\right.$. there is an $\left.s \in D_{e}, g(s)=1\right]\left(\left(\lambda q \in D_{e} . q\right.\right.$ is silly in $w$ according to perspective $p l$ and is a prank in $w))=\left[\lambda a \in D_{e} . \lambda b \in D_{e}\right.$. $b$ played $a$ in $w$ ] (there is an $s \in D_{e}$ such that $s$ is silly in $w$ according to perspective $p l$ and is a prank in $w)=\lambda b \in D_{e}$. $b$ played at thing that is silly in $w$ according to perspective $p 1$ and is a prank in $w$. 
Going back to the main thread, we get:

[[Johnny played a silly ${ }^{1}$ prank and got some tasty ${ }^{2}$ licorice] $]^{c, w}$, $<p 1, p 2, \ldots p n>=\left[\left[\lambda h \in D_{<e, t>} . \lambda i \in D_{<e, t>} . \lambda r \in D_{e} . h(r)=\right.\right.$ 1 and $i(r)=1]\left(\lambda y \in D_{e} . y\right.$ got a thing that is tasty in $w$ according to perspective $p 2$ and belongs to the kind licorice in $w)\left(\lambda b \in D_{e} . b\right.$ played at thing that is silly in $w$ according to perspective $p 1$ and is a prank in $w)]$ (Johnny) $=\left[\lambda r \in D_{e} . r\right.$ got a thing that is tasty in $w$ according to perspective $p 2$ and belongs to the kind licorice in $w$ and $r$ played at thing that is silly in $w$ according to perspective $p l$ and is a prank in $w$ ] (Johnny) $=$ Johnny got a thing that is tasty in $w$ according to perspective $p 2$ and belongs to the kind licorice in $w$ and played at thing that is silly in $w$ according to perspective $p l$ and is a prank in $w .^{34}$

\section{REFERENCES}

Anand, P., 2009, "Predicates of Taste Are Relativized to Kinds", presentation at SALT19, The Ohio State University, April 3-5, 2009.

Cappelen, H. and J. Dever, 2014, The Inessential Indexical: On the Philosophical Insignificance of Perspective and the First Person, Oxford University Press, Oxford.

Cappelen, H. and J. Hawthorne, 2009, Relativism and Monadic Truth, Oxford University Press, Oxford.

Cresswell, M., 1990, Entities and Indices, Kluwer Academic Publishers, Dordrecht.

- 1973, Logics and Languages, Methuen, London.

Egan, A., J. Hawthorne, and B. Weatherson, 2005, "Epistemic Modals in Context", in G. Preyer and G. Peter (eds.), Contextualism in Philosophy: Knowledge, Meaning, and Truth, Oxford University Press, Oxford, pp. 131-169.

Glanzberg, M., 2007, "Context, Content, and Relativism", Philosophical Studies, vol. 136, no. 1, pp. 1-29.

Heim, I. and A. Kratzer, 1998, Semantics in Generative Grammar, Blackwell, Oxford.

Kamp, H., 1971, "Formal Properties of 'Now'", Theoria, vol. 37, pp. 227274.

${ }^{34}$ I thank the audience at the Philosophical Analysis: Logic, Semantics, Metaphysics, Epistemology workshop, Slovak Academy of Sciences, Bratislava, 27.10.2017 and two referees for Critica for their suggestions, comments and criticisms. I acknowledge the financial help of a Lise Meitner grant (M2226-G24) from FWF. 
Kaplan, D., 1989, "Demonstratives”, in J. Almog, J. Perry and H. Wettstein (eds.), Themes from Kaplan, Oxford University Press, Oxford, pp. 481563.

Kindermann, D., 2019, "Coordinating Perspectives: De Se and Taste Attitudes in Communication", Inquiry, vol. 62, no. 8, pp. 912-955.

Kissine, M., 2012, "From Contexts to Circumstances of Evaluation: Is the Trade-off Always Innocuous?", Synthese, vol. 184, no. 2, pp. 199-216.

Kneer, M., 2015, Perspective in Language, PhD thesis, EHESS.

Kneer, M., A. Vicente, and D. Zeman, 2017, "Relativism about Predicates of Personal Taste and Perspectival Plurality", Linguistics and Philosophy, vol. 40, no. 1, pp. 37-60.

Kölbel, M., 2009, “The Evidence for Relativism”, Synthese, vol. 166, no. 2, pp. 375-394.

— 2004a, "Faultless Disagreement", Proceedings of the Aristotelian Society, vol. 104, no. 1, pp. 53-73.

— 2004b, "Indexical Relativism vs. Genuine Relativism", International Journal of Philosophical Studies, vol. 12, no. 3, pp. 297-313.

Lasersohn, P., 2016, Subjectivity and Perspective in Truth-Theoretic Semantics, Oxford University Press, Oxford.

- 2009, "Relative Truth, Speaker Commitment, and Control of Implicit Arguments", Synthese, vol. 166, no. 2, pp. 359-374.

— 2008, "Quantification and Perspective in Relativist Semantics", Philosophical Perspectives, vol. 22, no. 1, pp. 305-337.

- 2 2005, "Context Dependence, Disagreement, and Predicates of Personal Taste", Linguistics and Philosophy, vol. 28, no. 6, pp. 643686.

Lewis, D., 1980, "Index, Context, and Content", in S. Kanger and S. Ohman (eds.), Philosophy and Grammar, Reidel, Dordrecht, pp. 79-100.

- - 1979, "Attitudes De Dicto and De Se", Philosophical Review, vol. 88, pp. 513-543.

MacFarlane, J., 2014, Assessment-Sensitivity: Relative Truth and Its Applications, Oxford University Press, Oxford.

Ninan, D., 2010, De Se Attitudes: Ascription and Communication, Philosophy Compass, vol. 5, no. 7, pp. 551-567.

Rabern, B., 2012, "Propositions and Multiple Indexing", Thought: A Journal of Philosophy, vol. 1, no. 2, pp. 116-124.

Rabern, B. and D. Ball, 2019, "Monsters and the Theoretical Role of Context", Philosophy and Phenomenological Research, vol. XCVIII, no. 2, pp. 392-416.

Recanati, F., 2007, Perspectival Thought: A Plea for Moderate Relativism, Oxford University Press, Oxford.

Rey, D., 2016, In Defense of Implicit Times, $\mathrm{PhD}$ thesis, University of Barcelona <http://www.tdx.cat/handle/10803/398833> [retrieved: $31 / 03 / 2019]$. 
Richard, M., 2004, "Contextualism and Relativism", Philosophical Studies, vol. 119, no. 1-2, pp. 215-242.

Roeper, T., 2016, "Propositions and Implicit Arguments Carry a Default General Point of View. Acquisition Evidence against Relativism and Subjectivity", in C. Meier and J. van Wijnbergen-Huitink (eds.), Subjective Meaning: Alternatives to Relativism, De Gruyter, Germany, pp. 210226.

Snyder, E., 2013, "Binding, Genericity, and Predicates of Personal Taste", Inquiry, vol. 56, no. 2-3, pp. 278-306.

Stalnaker, R., 1981, "Indexical Belief”, Synthese, vol. 49, no. 1, pp. 129 149.

Stephenson, T., 2007, "Judge Dependence, Epistemic Modals, and Predicates of Personal Taste", Linguistics and Philosophy, vol. 30, no. X, pp. 487-525.

Stojanovic, I., 2012, “The Problem of De Se Assertion”, Erkenntnis, vol. 76, no. 1 , pp. 49-58.

Torre, S., 2010, "Centered Assertion", Philosophical Studies, vol. 150, no. x, pp. 97-114.

Vlach, F., 1973, "Now" and "Then": A Formal Study in the Logic of Tense Anaphora, PhD thesis, UCLA.

Weber, C., 2013, "Centered Communication", Philosophical Studies, vol. 166, supplement 1, pp. 205-223.

Zeman, D., 2018, "Perspectival Plurality, Relativism, and Multiple Indexing", in R. Truswell, C. Cummins, C. Heycock, B. Rabern and H. Rohde (eds.), <https://semanticsarchive.net/Archive/DRjNjViN/Zeman.pdf > Proceedings of Sinn und Bedeutung 21, vol. 2, pp. 1353-1370.

— , 2015, "Relativism and Bound Predicates of Personal Taste: An Answer to Schaffer's Argument from Binding", Dialectica, vol. 69, no. 2, pp. 155-183.

Received: August 15, 2017; revised: September 8, 2018; accepted: February 6, 2019. 